\title{
THE CHEMICAL \\ ORGANIZATION OF LIVING MATTER
}

C. F. KRAFFT

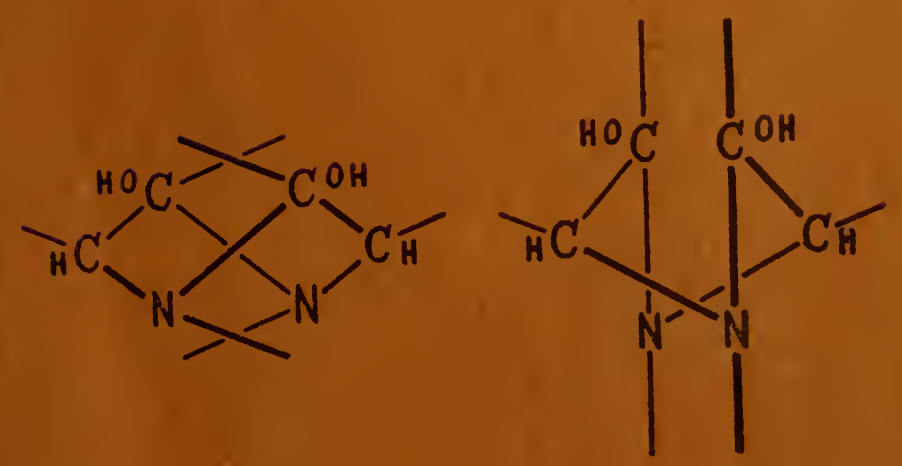

\author{
(opyright 1938 \\ by Carl i'. KRaffT
}

QH

335

K 84

c. 2 
Life is purely a physical phenomenon. All the phenomena of life depend on mechanical, physical, and chemical causes, which are inherent in the nature of matter itself. The simplest animals and the simplest plants, which stand at the lowest point in the scale of organization, have originated and still originate by spontaneous generation.

—Jean Lamarck, 1809. 


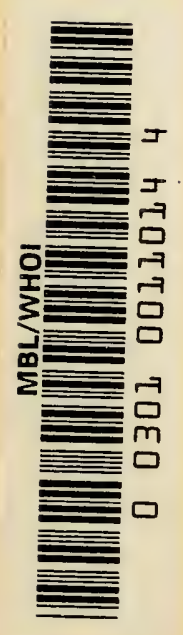



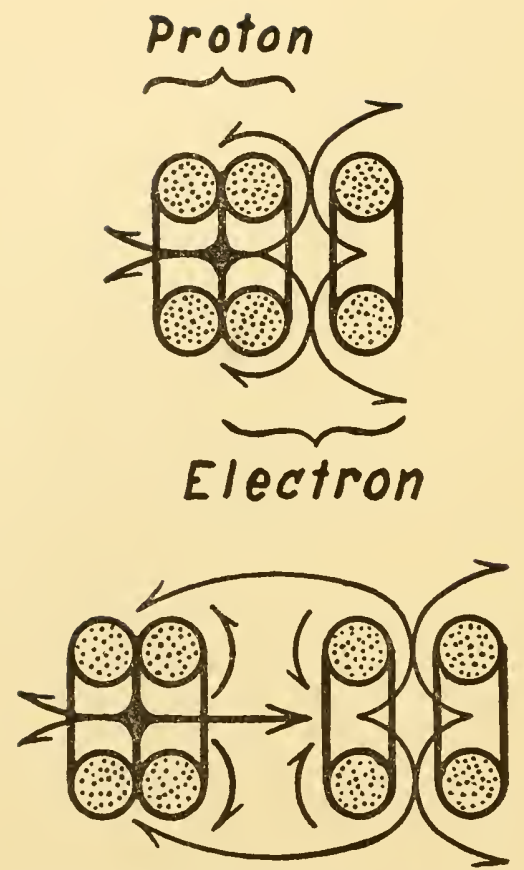

Neutron (above) and Hydrogen Atom (below)

Copyright 1937 by Carl F. Krafft 


\title{
THE CHEMICAL ORGANIZATION OF LIVING MATTER*
}

\author{
Carl F. Kraffit \\ Copyrights 1927, 1937, and 1938 \\ by Carl F. Krafft.
}

\section{INTRODUCTION}

The question whether the chemical organization of living matter is such as can adequately account for life processes is at the basis of every. argument between vitalism and mechanism. A few decades ago the issue was clearly defined, and anyone who had any opinion at all in the matter was definitely on one side or the other. Today, however, the situation is chaotic. The efforts of the reconcilers have only resulted in confusion. Instead of a clearly defined issue between vitalism and mechanism, we now have "holism" and "organicism"' whatever these may mean. It brings to mind the old saying of Mephistopheles: "Denn eben wo Begriffe fehlen, da stellt das Wort zur rechten Zeit sich ein." (For just where concepts are lacking, there the word introduces itself at the opportune time.) It may confidently be stated, however, that the thoughtful student of modern science will never be satisfied with any "reconciliation" which does not give a definite answer of "yes" or "no" to the question whether there exists in the living organism a metaphysical entity in addition to the chemical elements C, H, N, O, S, P, etc., and their structural organizations.

* Presented before the International Congress of Plasmogeny and General Culture, Mexico City, July, 1938. 


\section{ATOMIC STRUCTURE AND THE LIVING ORGANISM}

Living organisms, both unicellular and multicellular, have many characteristics which are similar to those of the elementary atoms-to wit their localized individualities which they maintain in spite of a changing environment, their striving to complete their structures according to predetermined patterns, their spontaneity of action and arbitrariness of behavior, their symmetrical and centralized organizations, and their rounded contours.

Another property which living organisms probably have in common with the atoms of matter is the possession of mind and consciousness. In the higher animals consciousness originates in the central nervous system, and is probably nothing else than the subjective aspect thereof. Now what is there in the structure of the central nervous system that causes it to have consciousness? It can be nothing other than its centralized organization - some peculiar system of coordination which causes the entire system to function as a unitary and indivisible entity. But if centralization of structure produces consciousness in the central nervous system, then there should be consciousness wherever there is a similar centralization of structure, as for example in the atom. Since it has been shown by the researches of P. Lenard and $\mathrm{E}$. Rutherford that the elementary atoms have centralized structures, it may reasonably be assumed that they also possess consciousness. (Centralization of structure is all that was actually proved by the deflection experiments on which the nuclear theory of atomic structure was based. The existence of an atomic nucleus containing within it the entire mass of the atom is merely a gratuitous assumption.)

Since the living organism has so many features in common with the atom, it appears that the study of pro- 
toplasmic structure should not be entirely dissociated from the study of atomic structure. The same dynamic "ether" which must be assumed to circulate through each atom of matter to bind its parts together probably also circulates through the protoplasmic system of every living organism so as to give it centralized control over biological activities, and unity of consciousness.

It is not so much the prevailing nuclear theory of atomic structure which is significant in this respect, nor the classical vortex theory of the 19th century, but rather the new vortex theory which was first introduced by Hermann Fricke in Berlin, and was later developed in detail by the writer (C. F. Krafft in Washington, D. C.). The classical vortex theory of the 19th century was a failure because it tried to proceed with the erroneous assumption that the ether is entirely frictionless and that adjacent vortex filaments would have no effect upon one another. The new vortex theory of Fricke and Krafft assumes that the ether has quasi-frictional properties by virtue of which not its energy but its direction of flow is affected. More specifically, this new vortex theory assumes that adjacent vortex filaments must have rolling contact in order to form stable configurations.

A complete presentation of this new vortex theory cannot be attempted here, but certain details will have to be explained because of their relation to protein chemical structure. It is the structure of the nitrogen atom and the form of the valence bond that we are particularly concerned with in the study of living matter. The new vortex theory has shown that the nitrogen atom always has three primary valence bonds, not uniformly distributed, but all on one side of the atom-a fact which has been established independently on the basis of purely experimental evidence. Tetravalent and pentavalent nitrogen are produced by branching or bifureation of one or the other of these primary valence bonds. In quanti- 
tative chemical analysis this new nitrogen atom is in every respect the equivalent of the nitrogen atom of the nuclear theory, but in protein chemical structure where we are concerned with the spatial positions of the atoms, it leads to entirely different structural patterns.

\section{POLYPEPTIDE SPIRALS}

Prior to 1927 we knew nothing about protein chemical structure which served in any way to clarify life processes. It appeared from the researches of Emil Fischer and his collaborator's that proteins consisted principally of polypeptide chains or diketopiperazine rings, but there was no suggestion as to how these were arranged in space or how they were produced in the living organism. The prevailing opinion was that the chemical structure of living matter is so complex that its spontaneous formation by the fortuitous play of natural forces could have occurred only once in eons, and that it would be folly to attempt to produce such structure synthetically. These views have changed considerably since then. It is now realized that the complex chemical structures which must constitute the hereditary patterns of the higher plants and animals have developed gradually in the course of evolution, and probably do not occur in the simplest unicellular organisms. Life in its broadest and simplest aspects is nothing more than self-perpetuation, and the mechanism that is necessary for this purpose need not be any more complex than the "self" that is being perpetuated. As explained by the writer in his 1927 monograph Spiral Molecular Structures, The Basis of Life, the method that nature uses in the perpetuation of any hereditary pattern, whether simple or complex, is probably nothing other than the obvious geometric scheme of confining the pattern to two dimensions of space, so as to leave the third dimension available for the perpetuation of this pattern. In fact, it is 
inconceivable how heredity could be accomplished by any other method, and yet there is not a textbook of biology in existence which contains a clear statement of this proposition! On the contrary, as late as 1935 we read in The Philosophy of a Biologist by J. S. Haldane the following disheartening statement:

We can form no conception on these lines [of the prevailing mechanistic conception of physics and chemistry] of how it is that a living organism, presuming it, as we must on the mechanistic theory, to be an extremely complex and delicately adjusted piece of molecular machinery, maintains and adjusts its characteristic form and activities in the face of a varying environment and reproduces them indefinitely often. (p. 37.)

It will not be necessary to make any lengthy comments on the above quotation of Haldane, because the very language he uses shows a deplorable lack of familiarity with modern scientific concepts. No present-day mechanist tries to maintain that the living organism is merely a piece of "molecular" machinery. There are several other types of chemical structure besides the "molecular" structure, and in living matter it is principally the nonmolecular structures which control biological activities.

Statements that science cannot explain this or that on a mechanistic basis, although seldom made by scientists, are frequently heard emanating from the pulpits. Since statements of that sort constitute direct attacks upon science, it becomes not only the privilege but also the duty of scientists to make their reply. The fallacy of all such statements about the supposed limitations of science lies in the fact that living matter, as it exists in the cells of the higher animals, is itself complex beyond comprehension, and therefore cannot be said to lack the necessary complexity to account for the manifold activi- 
ties of the living organism. As will be seen later, the polypeptide spiral which probably constitutes the ultimate unit of living matter measures only about 5 Angstrom units in width, whereas the nuclei of the cells of the higher animals are about 3000 times larger. A single germ-cell of one of the higher animals may therefore contain many thousands of polypeptide spirals. If now we assume that each spiral can be attached to the one immediately preceding it in either of two different ways, then the number of different patterns which would be theoretically possible would be 2 multiplied by itself thousands of times-and this represents only that portion of one's makeup which is inherited. The real complexities do not begin until this inherited pattern is elaborated in millions of different varieties in the cells of the cerebral cortex. Let no one say that the mechanistic conception of life is inadequate to account for the richness of variety in our conscious experience.

The polypeptide system of protein chemical structure, (and also the diketopiperazine system, as will be explained later,) will readily adapt itself to the above-mentioned geometric theory of heredity. By arranging a number of polypeptide chains in parallelism, and connecting them to one another through side-chains, we can produce structures having any desired pattern in cross-section, but with the longitudinal dimension remaining available for the perpetuation of this pattern by endwise growth of the polypeptide chains. In reality these polypeptide chains are probably not rectilinear but in the form of helical spirals with six atoms to a convolution. Such a spiral structure would also account for the optical activity (rotation of the plane of polarization of light) which is always exhibited by amino acids obtained from natural sources.

The parallelism of the polypeptide chains in naturally occurring fibrous proteins (silk fibroin, keratin, myosin, 
and collagen) has been verified experimentally by the X-ray diffraction studies of W. T. Astbury and others. The globular and crystalline proteins (egg albumin, haemoglobin, edestin, insulin, and pepsin) will not lend themselves so readily to the same methods of investigation, but their similarity in chemical constitution and properties to the fibrous proteins makes it reasonable to assume that they have fundamentally the same chemical structure. This conclusion is corroborated by a comparison of the identity intervals found in the X-ray diffraction patterns of these two classes of proteins. As stated by Astbury (Proc. Roy. Soc. London, A 150, p. 549, 1935) :

There would appear to be a great gulf between the molecular structure of feather keratin and that of crystalline pepsin, yet-unless this is nothing more than a remarkable coincidence-the X-ray photograph of the former reveals a striking analogy with that of the latter. The principal longitudinal and lateral periodicities found in the structure of feather keratin are in close relation to the corresponding periodicities of unaltered crystalline pepsin.

It is also necessary for an intelligent interpretation of biological processes to assume that the protein constituents of fluid protoplasm have a fibrous, and not merely a globular constitution. In his article On the Structural Framework of Protoplasm (Scientia, July 1, 1937, p. 7,) R. A. Moore states:

Since protoplasm, as distinguished from nucleus, is endowed with specific characters it seems reasonable to suppose that they have a structural basis, and that living matter is a more ordered and complicated thing than a chaos of particles in a fluid matrix such as an emulsion or suspension. ... The dynamic facts require building stuff with directional possibilities, the forerunners of structure. We may suppose that the protoplasm contains polar particles or chains of molecules, which, when occasion arrives, 
link themselves together to form structures, in somewhat the fashion in which micells of cellulose are joined together to form a cell wall.

It is inconceivable how heredity or self-perpetuation can be accounted for on the basis of anything except a fibrous chemical structure, and since we know of at least one crystalline or globular protein (tobacco leaf virus) which does exhibit heredity and self-perpetuation, we are compelled to assume that a fibrous chemical structure is present in at least some of the globular proteins.

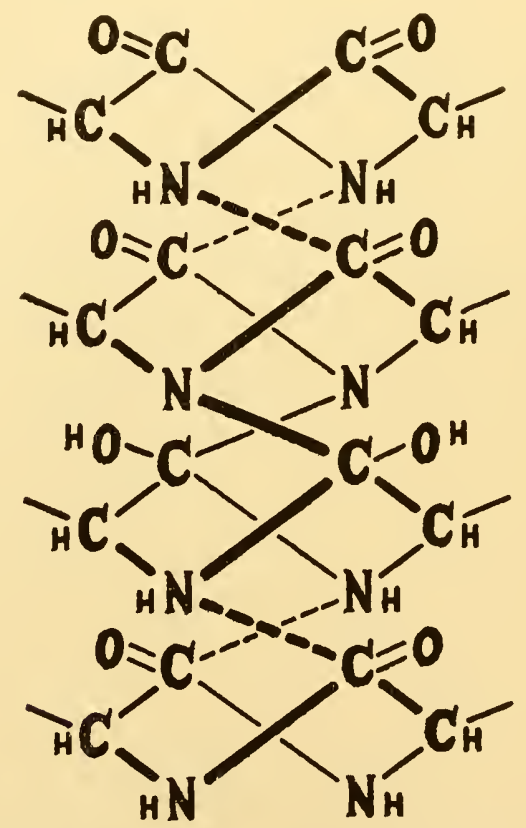

Double-strand polypeptide spiral. The diketopiperazine ring at the upper end is in the process of assimilation, while the one at the lower end may be considered as being in a state of temporary attachment to a proteolytic enzyme.

Every living organism is so constituted that growth by assimilation of amino acid residues can take place independently of other physiological processes. Since it must 
be assumed that physiological processes depend in some way on the chemical activity of living matter, one would think that such processes would be disturbed or disrupted whenever additional amino acid residues are assimilated. Thus in the spiral polypeptide structure as proposed in 1927, assimilation would have to take place by the addition of one amino acid residue after another so as to build up the spiral by successive increments of half a convolution at a time. This would bring the free end of the spiral alternately to diametrically opposite sides, which would produce a continual change in the external chemical configuration at the free end of a cluster of spirals. Such a continual fluctuation in the chemical configuration of living matter would seriously interfere with physiological functioning. A satisfactory way out of this difficulty was found in 1937 when it was discovered that the polypeptide spiral may consist of two polypeptide chains instead of one, so that the assimilation of amino acid residues can take place simultaneously at diametrically opposite sides of the spiral. (Life a Vortex Phenomenon, p. 7 ; The Scientific Press, Aug. and Dec., 1937.)

\section{MAGNETISM AND LIFE}

The phenomenon of magnetism probably plays an important part in life processes. The adaptability of magnetism for simulating the fundamental biological and biochemical processes has been frequently commented upon, but until recently it has never been seriously proposed as the real cause of such processes, for the obvious reason that living matter shows no response to a magnetic field. However, a piece of glass (or any other transparent material) is also not magnetic, and yet when it is placed in a magnetic field it will rotate the plane of polarization of light, although neither the glass alone nor the magnetic field alone will produce any rotation. The magnetic field here coacts in some manner with the atoms or molecules 
of which the glass is composed. This proves that magnetism can act upon matter in other ways than that with which we are all familiar. A magnetic field being nothing but a whirlwind in the ether, it is reasonable to assume that any twisted chemical structure will produce a miniature whirlwind of this sort. The mere existence of an ether whirlwind of molecular dimensions is not sufficient, however, to constitute life. The chemical structure which maintains this whirlwind must be so constituted that it will combine chemically with additional structural units along the axis of the whirlwind, and still keep the same twisted configuration at the free end. Furthermore, in order to produce the complex hereditary patterns of the higher plants and animals, or to produce any kind of structure large enough to be visible under the microscope, it is also necessary that these twisted chemical structures be capable of connection to one another laterally along at least three different sides. The polypeptide spiral (and also the sulphur-peptide spiral as we shall see later) will satisfy all of these conditions.

The fact that living matter does not respond to a magnetic field in the same manner as a piece of iron is no disproof of this magnetic theory of life, because in a magnetized piece of iron the north and south poles are at an appreciable distance apart, and the force exerted upon it by a magnetic field depends on this distance. In a polypeptide spiral, however, the distance between opposite ends is extremely small, so that a magnetic field could not act upon it with any appreciable force.

A magnetic whirlwind around the axis of a polypeptide spiral should exert a coordinating effect upon any amino acid residues in its immediate vicinity, and if two such residues are simultaneously present, they will arrange themselves around the axis of the whirlwind in the form of a diketopiperazine ring. With the ring held in this position, it can be readily joined to the end of the spiral 
by direct union between the $-\mathrm{CO}-$ groups at the end of the spiral and the - NH - groups of the newly formed diketopiperazine ring. The two hydrogen atoms of the imide groups of the newly formed diketopiperazine ring will probably be taken over by the oxygen atoms at the end of the spiral so as to form hydroxyl groups, which will leave the nitrogen atoms of the diketopiperazine ring free to combine with the hydroxylated carbon atoms at the free end of the spiral. After assimilation of each new diketopiperazine ring, the configuration of atoms at the end of the spiral will be the same as it was before, the spiral having been merely increased in length.

The theory that life is a magnetic phenomenon is quite old, but we had no experimental verification of this theory until 1935 when W. D. Francis of the Botanic Gardens in Brisbane, Australia, published his pamphlet on Iron as the Original Basis of Protoplasm-The Generation of Life in Space and Time. For many years Francis has been conducting experiments on the synthesis of proteins by means of metallic iron in the presence of inorganic salts and atmospheric carbon dioxide, but in 1935 he discovered that the ferrous hydroxide which was produced in his experiments was definitely magnetic. He therefore concluded that

In view of the ultimate origin of protoplasm in iron it is quite likely that magnetic properties perform a much more prominent part in life processes than is realized at present. (p. 10.)

Two subsequent pamphlets were published by Francis in 1936 and 1937 in which he presented additional observations on the magnetic properties of ferrous hydroxide and the iron bacterium Leptothrix. He also found evidence of a spiral structure in the protoplasmic materials under observation, and in a recent communication to the writer he stated that 
It appears practically certain that the fundamental structures of all living organisms are spiral (helical).

And yet there is not a textbook of biology in existence which even mentions the spiral chemical structure!

\section{PROTEIN CHEMICAL FABRIC}

In order to account for the complex hereditary patterns of the higher plants and animals, we must assume that there are at least three points on the periphery of each spiral where it can be attached to adjacent spirals. One mode of attachment is undoubtedly between two hydroxyl groups by the elimination of a molecule of water, the two spirals being then connected through intermediate oxygen atoms. It is interesting to observe that this comnection is similar to that which occurs in polysaccharides.

The hydroxyl groups, however, occur only at two diametrically opposite sides, so that connections through oxygen atoms could produce only flat sheets but not three-dimensional patterns. There must be some other points of connection, but where can they be? The carbon atoms have all their valencies occupied, and the three valencies of the nitrogen atoms are also occupied. The additional connections must be in the form of branched valence bonds, such as we have in tetravalent and pentavalent nitrogen, and will be most likely to occur between the nitrogen atoms and the alpha carbon atoms (those of the - CHR- groups) so as to produce something in the nature of hydrogen bonds. With these two kinds of bonds we can produce rectangular lattice structures as shown in the diagram. Since the distance between adjacent carbon and nitrogen atoms in organic compounds is about 1.4 Angstrom units, we can calculate the dimensions of the rectangular compartments of this 

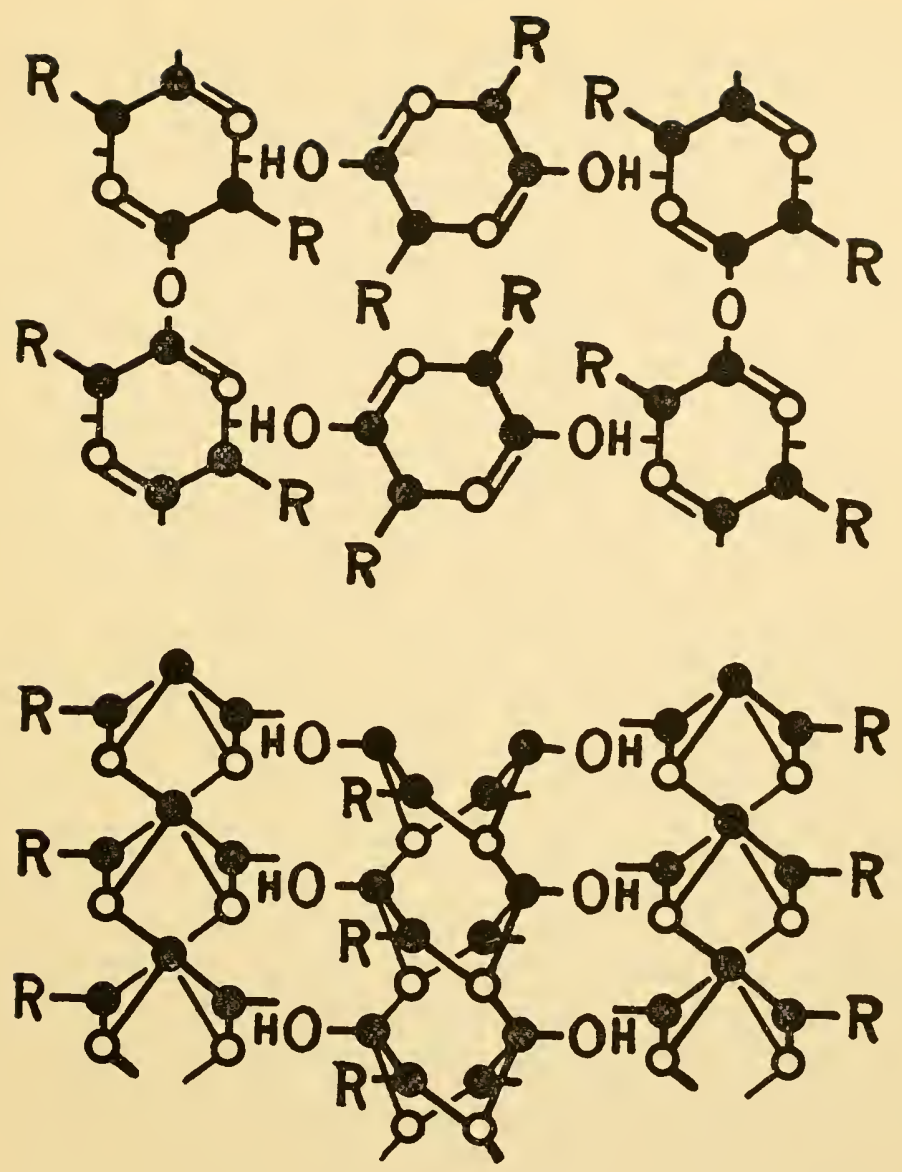

Protein chemical structure in plan view (above), and in side view (below). Black dots represent carbon atoms (and also sulphur atoms in case of sulphur-peptides), and small circles nitrogen atoms.

lattice, and will find them to measure approximately 4.5 $\mathrm{x} 10$ Angstrom units, which is in perfect agreement with the identity intervals found in X-ray diffraction patterns of natural proteins.

It will be seen from the diagrams that the protein chemical structure has a polarity somewhat similar to that of a magnet, and may actually consist of a cluster of miniature molecular magnets. Such a structure will be 
alkaline at one end and acid at the other end, and if division occurs, each of the fragments will have a similar polarity. Assimilation of amino acid residues probably occurs at the hydrophilic carboxyl ends, but as these may be located elsewhere than on the periphery of the cell, the problem presents itself of getting the necessary food material to them, especially when the food material is not soluble in the surrounding medium. This difficulty is overcome by the liberation of small fragments or "enzymes" from the living cell, which will be free to migrate out to the food material and make their attack upon it, setting free the amino acids. There seems to be no reason for assuming that these amino acids are actually transported by the enzymes into the living cells. In fact, the ability of minute traces of proteolytic enzymes to digest almost unlimited quantities of dead protein material seems to indicate that these enzymes do not remain attached for any length of time to the fragments which they dislodge. Neither does there seem to be any basis for the recent suggestion that enzymes act as organizers for the intracellular proteins. Such a hypothesis merely substitutes a greater difficulty for the lesser one. As explained by the writer in his 1927 monograph Spiral Molecular Structures, the Basis of Life, proteins are so constituted that they act as their own organizers.

If proteolytic enzymes are broken-off portions from living cells, then they should have an acid-alkaline polarity and a cross-sectional pattern similar to that of the intracellular proteins from which they were derived. If the proteins of the food material have a similar pattern, or one which will permit sufficiently close approach of the enzyme molecules, then a temporary union of the enzyme with the dead food material may be assumed to take place, followed by dislodgement of some of the amino acid residues. Chemically active side-chains are parts 
of the molecular structure of every enzyme and determine the specificity thereof. Side-chains of one sort or another are probably attached to all sides of the protein portion of the enzyme molecule, but those which are at the amino end are the ones which are responsible for proteolytic activity if the enzyme is one which attacks the carboxyl ends of the food molecules. In the pepsin molecule it appears to be the tyrosine group which contributes such activity. (SCIEnce, Nov. 26, 1937, p. 482.) Although the specificity of an enzyme depends primarily on the chemical structure of some active side-chain, it is the normal protein portion which determines the degree of activity.

Those who wish to make a further study of the mechanism of proteolytic enzymes should read the article by Max Bergmann in the May 18, 1934 issue of Science. Experiments with peptide-splitting enzymes have shown that the presence of alpha hydrogen atoms in the amino acid residues, and their arrangement in the cis-position in diketopiperazine rings, are essential for the action of those enzymes which attack the carboxyl ends of polypeptides, although not for those which attack the amino ends. This seems to indicate that in the protein molecules the hydrocarbon side-chains (attached to the alpha carbon atoms) are slanted towards the amino ends and not towards the carboxyl ends of the spirals. Since these hydrocarbon side-chains are hydrophobic, they will be pressed back towards the other end of the protein molecule by repulsion from the water clinging to the hydrophilic carboxyl groups.

The subject of protein structure should not be concluded without a brief mention of monomolecular films, a preliminary account of which will be found in the Jan. 15, 1937 issue of Science, and a more detailed account in the June 3, 1938 issue. Most of the experiments were conducted with egr albumin, although it may be ex- 
pected that other proteins will show a similar behavior. In these experiments it has been shown that a dry monomolecular film of egg albumin is 20 Angstrom units thick, and is hydrophilic on one side and hydrophobic on the other side. It behaves like a two-dimensional network in which the units are connected by strong elastic springs. All of this is in perfect agreement with the writer's system of protein structure. If we accept the recent reports of chemists that the molecule of egg albumin has 288 amino acid residues (Journ. Brol. Chem., 118, 301, 1937), and that they are arranged in groups of 18 , then the entire molecule will consist of a cluster of nine doublestrand polypeptide spirals forming two rectangular compartments, each measuring approximately 4.5 x $10 \mathrm{Ang}-$ strom units. Each of these double-strand spirals will consist of a column of 16 superimposed diketopiperazine rings. When the material is thoroughly dried, these may he assumed to be closely nested upon one another, and will then form a column exactly 20 Angstrom units high. Since natural amino acids are always of the same optical activity (usual left-handed) it must be assumed that the hydrocarbon side-chains along the sides of the spirals always slant in the same direction, so that the doublestrand spirals, and in fact the entire protein molecule, will be definitely hydrophilic at one end (the carboxyl end), and hydrophobic at the other end (the amino end). Consequently when these molecules are all arranged with the same ends up, they will form a monomolecular film which will be hydrophilic on one side and hydrophobic on the other side. It will also be seen from the diagrams that the protein molecule has exposed rows of hydroxyl groups along the corners. These will form chemical bonds with the sides or corners of adjacent molecules, there being several points on the periphery of each molecule where such bonds could be formed. Since the molecules will have a random distribution in the plane of the 
film, their arrangement will be "smectic" (as distinguished from crystalline) so as to leave irregular open spaces between them. This accounts for the lateral compressibility of the film.

Besides carbon, nitrogen, oxygen, and hydrogen, there are also present in living matter a number of other ele. ments in lesser amounts, especially sulphur and phosphorus. These have not been considered hereinabove because they are not present in sufficient amounts to constitute regular structural elements of the protein chemical fabric. In a typical protein there is only about one sulphur or phosphorus atom to every hundred nitrogen atoms, whereas in order to constitute regular building units of polypeptide spirals there would have to be about cne sulphur or phosphorus atom to every one nitrogen atom. Sulphur seems to be normally present in the cytoplasm, and phosphorus in the nucleus. The experiments of F. S. Hammett at Wistar Institute have shown that the rate of cell division depends on the state of oxidation of the sulphur. The presence of phosphorus seerns to be necessary for the assimilation of amino acid residues, from which it appears that the phosphorus acts as a catalyst.

\section{SYNTHETIC LIFE}

One of the most important questions which confronts science today is whether we know enough about the chemical structure of living matter to justify us in making serious attempts to produce life synthetically. The authorities of today do not consider our attempts in this direction as being worthy of publication, but let it be remembered that the authorities of a hundred years ago also tried to maintain a similar attitude towards organic compounds, until they were abruptly interrupted by Wohler's synthesis of urea. The prevailing opinion today is that the living organism is too complex to be pro- 
duced by chemical synthesis, which is undoubtedly true of the large majority of living species, but may not be true of the simpler bacterial forms of life, such as the iron and sulphur bacteria and the amoebae. Recent experiments with tobacco leaf virus seem to show that life in its simplest forms is a function of certain kinds of protein molecules, and we have direct experimental evidence in the identity intervals of $\mathrm{X}$-ray diffraction patterns that the ultimate structural units in protein molecules cannot be much more complex than the ordinary molecules of organic chemistry. In fact, from what has already been explained, it appears that the protein molecule is but little more than a polymerization system of diketopiperazine rings. The stability of proteins against heat and chemical treatment is also indicative of a comparatively simple chemical structure, the proteins being merely denatured or coagulated but not destroyed unless the treatment is very severe.

Although there seems to be no valid reason why it should not be possible to produce living matter by chemical synthesis, nevertheless there exists an emotional prejudice against all efforts in this direction, which is probably the reason for such a deplorable scarcity of accredited experimental investigation-and what is still more unfortunate, this prejudice does not come exclusively from the laity, but also from scientists of high standing. It is therefore not surprising that there has been so little progress in this important branch of science, because scientists can hardly be expected to spend their time and energy on enterprises which would precipitate upon them the wrath and ridicule of those on whom they must depend for their livelihood, especially when the results of their investigations would probably be denied publication in any of the accredited scientific magazines. It will not be necessary to make any comments as to where this prejudice originates. As stated by Oscar Riddle of 
the Carnegie Institution in his Jan. 1, 1936 address before the American Association for the Advancement of Science :

The present restrictive influence of organized religion on the teaching of the best of biology is intolerable. (Scinnce, Jan. 24, 1936.)

Notwithstanding these unfavorable conditions, there have been a few investigators who have done considerable work on this problem during recent years, and foremost among these have been A. L. Herrera in Mexico, and W. D. Francis in Australia. Although both of these investigators have been working on the same problem, they have tried to solve it by entirely different methods, and with widely different results. Herrera used $36 \%$ formaldehyde solution (3 grams) as the principal source of carbon, whereas Francis used atmospheric carbon dioxide. Herrera formerly used ammonium sulphide with oxides of nitrogen, and more recently ammonium thiocyanate (1.5 grams) as the somree of nitrogen and sulphur, whereas Francis used the following mixture:

$\begin{array}{ll}\text { Ammonium sulphate } & 1.50 \text { grams } \\ \text { Potassium chloride } & 0.05 \text { 66 } \\ \text { Magnesium sulphate } & 0.05 \text { 6 } \\ \text { Potassium monohydrogen phosphate } & 0.05 \text { "6 } \\ \text { Calcium nitrate } & 0.01 \text { liter } \\ \text { Distilled water } & 1.00 \text { liter } \\ \text { Metallic iron wire } & \end{array}$

Herrera obtained a multitude of life-like and actively moving organisms closely resembling natural protozoa and exhibiting in every detail the phenomenon of mitotic cell division, whereas Francis did not obtain any actively moving organisms or anything resembling natural protozoa. On the other hand Francis claims to have obtained synthetic proteins whereas Herrera has found only amino acids and starch. 
As far as can be determined from his published reports, the experiments of Francis were conducted with the most rigorous precautions against accidental contamination, and the identification of his synthetic products as proteins was based upon all the usual chemical tests. The same experiments conducted in the absence of atmospheric carbon dioxide did not result in the formation of proteins. Francis expressed the opinion that the synthesis of proteins was initiated on the surfaces of the ferrous hydroxide granules, and that it was in some way dependent on the magnetic effect of these granules-a speculation which seems quite plausible from the standpoint of the spiral polypeptide theory. On the other hand it must be remembered that iron silicates are sometimes formed under similar conditions, and look very deceptive. The experiments of Francis should be carefully repeated so as to determine definitely whether he had really produced proteins, or only iron silicates.

Although the synthetic production of proteins, if it can be verified by others, would be a long step ahead in the direction of synthetic life, yet it should not be looked upon as the final solution of the problem. This was admitted by Francis when he stated in his 1932 pamphlet that

One of the most prominent features of an organism, which is absent from the specks and patches of ferruginous material, is specific form. (p. 36.)

In the absence of any specific forms there can also be no cell division, whereas in the preparations of Herrera there has been an abundance of cell division. Francis, however, considers cell division not absolutely essential in the lowest forms of life:

The primal forms of life are visualized as composed largely of mineral constituents, such as ferrous hydroxide. The ability of these precursors of 
organisms to generate highly complex compounds, such as protein, indicates their relationship to the organisms recognized by the biologist. It can be contended that these alleged early forms do not possess many of the characteristics of organisms, as for instance the power of reproduction. As, however, they are derived directly from inorganic material, there is scarcely any necessity for the ability of reproduction. They are generated directly from the simple compounds of rocks, soils, water and the atmosphere. Their requirements and equipment are, therefore, not always identical with these of the organisms recognized by the scientist. (p. 37.)

The experimental methods of Francis, unlike those of Herrera, did not lead to the production of starches:

I have no idea as to what intermediate products were formed during the combinations leading to the eventual generation of protein. Apparently no starch was present in the preparations tested for protein with iodine as its presence would have been indicated by the familiar blue color. (p. 34.)

The experiment showing that protein bodies are formed when the solution with the suspended iron wire was kept in darkness shows conclusively that the production of protein is independent of light. This experiment and the fact that the primitive nitrite and nitrate bacteria assimilate the carbon dioxide of the air in darkness suggest that this process, known as chemosynthesis, is more fundamental than the photosynthesis effected by green plants. In the vital processes functioning at the earth's surface, the energy of sunlight (photochemical energy) may. displace, to some extent, the energy of mineral compounds in a reduced state, especially as mineral compounds are more highly oxidized towards the surface than lower down towards the unaltered rocks. (p. 37.)

With only carbon dioxide as the raw material, and in the absence of chlorophyll, the formation of starches 
could hardly be expected, even in the presence of sunlight. On the other hand in the experiments of Herrera it is possible for the formaldehyde $\mathrm{CH}_{2} \mathrm{O}$ to polymerize into formose $\mathrm{C}_{6} \mathrm{H}_{12} \mathrm{O}_{6}$, which differs from starch $\mathrm{C}_{6} \mathrm{H}_{10} \mathrm{O}_{5}$ only by an extra molecule of water.

If proteins were actually produced in the experiments of Francis, then the carbon of such proteins must have come from the carbon dioxide by partial reduction to formaldehyde at the surface of the iron or ferrous hydroxide. The amino nitrogen could have come either from the ammonium ions or from the nitrate ions. Francis may have obtained the spiral polypeptide structure, but it is doubtful whether there was any reduction of the sulphate to sulphides, with the formation of cystine or cysteine.

In the experiments of Herrera it is possible that substances similar to proteins may have been produced, even though they were not detected by chemical tests. The polypeptide spiral as pictured hereinabove may be only one of several different structures that can be formed by spiral polymerization. There are other cyclic structures besides the diketopiperazine ring which have diametrically opposite acidic groups and also diametrically opposite basic groups so as to be capable of polymerization by superposition of the rings upon one another.

It has been found by Schmerda (Z. Angew. Chem., 30, 176,1917 ) that ammonium thiocyanate and formaldehyde in aqueous solution will combine, without any liberation of carbon dioxide, to form a soft yellow resin having no definite melting point, which is insoluble in all ordinary solvents, and which readily splits off formaldehyde when heated. Schmerda has shown that this resin contains twice as many nitrogen atoms as sulphur atoms, but apparently he did not make any carbon and hydrogen de- 


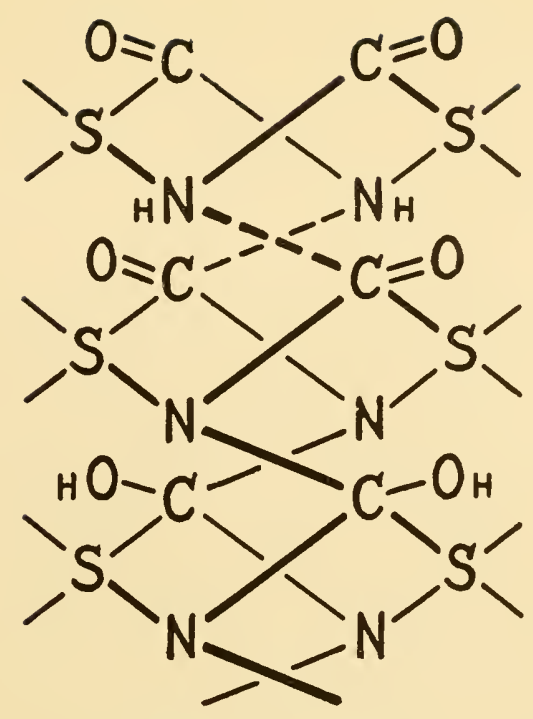

Sulphur-peptide spiral.

termination. He has suggested for it the following formula :

$$
\begin{aligned}
& \mathrm{N} \equiv \mathrm{C}-\mathrm{S}-\mathrm{NH}_{3}-\mathrm{CH}_{2} \mathrm{OH} \\
& \mathrm{HOCH}_{2}-\mathrm{NH}_{3}-\mathrm{S}-\mathrm{C} \equiv \mathrm{N}
\end{aligned}
$$

It is significant that the six-atom ring in this formula is exactly of the type that is needed for the building of peptide spirals, although no one at that time knew anything about spiral polymerization.

The first thing that probably happens upon mixing the two ingredients is a combination of the ammonium nitrogen with the formaldehyde carbon. At the same time there may be oxidation of the sulphur by atmospheric oxygen, and hydrolysis of the cyanide group, so as to produce the following intermediate product:

$$
\mathrm{NH}_{2}-\mathrm{CO}-\mathrm{SO}-\mathrm{NH}_{3}-\mathrm{CH}_{2} \mathrm{OH}
$$

If the basic $\mathrm{NH}_{2}-$ groups and the acidic - $\mathrm{SO}-$ groups of adjacent molecules then react with each other, 
ring structures similar to the one suggested by Schmerda may be formed:

$$
\begin{gathered}
\stackrel{\mathrm{H}}{\mathrm{N}}-\stackrel{\mathrm{O}}{\mathrm{C}}-\stackrel{\mathrm{OH}}{\mathrm{S}}-\mathrm{NH}_{3}-\mathrm{CH}_{2} \mathrm{OH} \\
\mathrm{HOCH}_{2}-\mathrm{NH}_{3}-\underset{\mathrm{HO}}{\mathrm{S}}-\mathrm{O}-\underset{\mathrm{H}}{\mathrm{H}}
\end{gathered}
$$

In the ring structures thus produced we have the same peptide group - $\mathrm{CO}-\mathrm{NH}$ - as in polypeptides, so that these rings should be capable of further polymerization into double-strand spirals by superposition upon one another in the same manner as diketopiperazines. In the double-strand sulphur-peptide spiral the chain-sequence $-\mathrm{N}-\mathrm{C}-\mathrm{S}-\mathrm{N}-\mathrm{C}-\mathrm{S}-$ will occur not only around the rings but also along the length of the spiral, just as in polypeptide spirals the sequence $-\mathrm{N}-\mathrm{C}-\mathrm{C}-\mathrm{N}-\mathrm{C}-\mathrm{C}-$ occurs not only circumferentially but also longitudinally.

It is believed that under the conditions of Herrera's experiments there will be some transformation similar to the one outlined above, rather than the formation of thiourea resin. Ammonium thiocyanate will not change into thiourea except at elevated temperatures, and the attachment of a formaldehyde group to the ammonium nitrogen will probably hinder, rather than help the formation of thiourea. On the other hand the hydroxyl group of the formaldehyde residue will be brought into close proximity with the cyanide group (on account of the angular relationships of the valencies) so as to assist in the hydrolysis of the latter.

In the sulphur-peptide spirals there will be rows of hydroxyl groups along diametrically opposite sides, just as in polypeptide spirals, so that the same type of lattice structure should be produced in both cases. This may be the reason why Herrera has obtained cell-structures and mitotic figures which closely resemble those of living cells. It is possible that sulphur-peptide spirals of this sort may have gradually changed over into ordinary poly- 
peptides in the course of evolution, or they may have been the precursors of the present sulphur bacteria.

There is a considerable difference in size between the polypeptide or sulphur-peptide spirals measuring less than one millimicron in diameter, and the smallest living organisms, such as tobacco leaf virus, measuring about fifty millimicrons in diameter. Organisms like tobacco leaf virus could not have been produced spontaneously by the fortuitous play of organic molecules. Either they have a long evolutionary ancestry of simpler organisms, or they are in the nature of enzymes which have separated from more complex organisms. If they were derived from simpler organisms, then there should be representatives of those simpler organisms in existence today, and with present-day experimental methods they would have been discovered if they existed. Actually, however, there is hardly a single nonparasitic free-swimming submicroscopic organism known. On the other hand if such virus organisms are degeneration-products of ordinary living cells, then we should look for the origin of life in the spontaneous formation of such cells.

It is at this point where the experiments of Herrera are of significance, for Herrera has shown that artificial cells produced from comparatively simple chemicals can exhibit all the familiar manifestations of unicellular life which are generally associated with living cells, even though the usual polypeptide structures may not be present. It appears from Herrera's experiments that life originated, not by the gradual building up of genes and complex hereditary patterns on a submicroscopic scale, but rather by the generation of tiny liquid vortices around granules of oxidizable material. All higher forms of plant and animal life derive their energy from oxidation of carbon compounds which were originally produced by photosynthesis in green plants. This photosynthetic process, however, requires the presence of chlorophyll which is a highly complex compound and could only have 
been produced after a long process of evolution. In the more primitive forms of life which existed before the appearance of chlorophyll, the energy necessary for carrying out life processes was probably derived, at least in part, from the oxidation of ferrous bicarbonate, ammonia, hydrogen sulphide, or elementary sulphur. The enormous deposits of bog iron ore probably originated in this manner. Now wherever there is a liberation of energy there is liable to be action of some sort, and it is conceivable that some of the energy resulting from this process of oxidation may have been spent in putting the surrounding fluid into circulation so as to generate small vortices. These, in turn, may have brought more ferrous bicarbonate into the presence of dissolved oxygen, and thus the process may have become self-sustaining.

Simultaneously with the oxidation of ferrous bicarbonate there may also have occurred a reduction of carbon dioxide to formaldehyde, which latter process may have been stimulated by the ultraviolet light from the sun. Iron compounds, in the presence of sunlight, will act photosynthetically in somewhat the same manner as chlorophyll. Any formaldehyde which may thus have been produced would then be likely to polymerize into more complex compounds containing chains of carbon atoms and in the presence of nitrogenous and sulphur compounds these may have organized themselves, first into irregular groups of sulphur-peptide or polypeptide spirals, and later into specific hereditary patterns.

The possible formation of sulphur-peptide spirals in Herrera's experiments does not exclude the possibility that ordinary polypeptide spirals may also be formed. It is known that formaldehyde will condense into formose consisting of a chain of - $\mathrm{HCOH}$ - groups with a free aldehyde (- $\mathrm{CHO})$ group at one end, which can be readily oxidized to a carboxylic group (- $\mathrm{COOH})$. Recent experiments with polysaccharides have shown that chains of $-\mathrm{HCOH}-$ groups will readily split off water and 
undergo internal oxidation and reduction, some of the carbon atoms going off as carbon dioxide while others are reduced to $-\mathrm{CH}_{2}-$ groups. Such internal oxidation and reduction was probably the process by which petroleum oil was formed from cellulose, the prevailing theory that it was formed from marine life being plainly untenable. This process of internal oxidation and reduction takes place spontaneously at moderate temperatures and pressures provided the medium is slightly alkaline, as in the presence of free calcium carbonate. If unsaturated fatty acids of the type $-\mathrm{CH}=\mathrm{CH}-\mathrm{COOH}$ are formed in this manner, and they are acted upon by ammonia, or by oxides of nitrogen followed by reducing agents, it is possible that alpha amino acids or their anhydrides (diketopiperazines and polypeptides) may be formed.

N. B.-Much prominence has been given during recent year's to a system of protein structure consisting of polypeptide chains folded into six-atom rings, with intermediate -CO-CHR-NHgroups between successive rings. Although this folded polypeptide structure is usually accredited to W. T. Astbury, it was published by the writer in 1930, and is covered by a 1930 copyright owned by the writer. Attention is directed to the diagrams on page 33 of the writer's book on SpIRAzines, disclosing this identical structure.

There have also been numerous articles on a "cyclol" system of protein structure in which the polypeptide chains are folded into triangular clusters with central triazine rings. Although this eyclol pattern is generally accredited to Dorothy M. Wrinch of Oxford, it was published by the writer in 1931, and is covered by a 1931 copyright owned by the writer. Attention is directed to the diagrams on pages 44 and 45 of the writer's book, "CAN ScIENCE ExPlatN LIFE?," disclosing the cyclol pattern in every detail.

The reader is therefore urged not to summarily reject this new spiral system of protein structure, just because it is not generally recognized in the authoritative literature of today. Those systems which are being recognized were originated by the writer, so that it should be the writer's privilege now to discard them in favor of a better system. 



\section{ELASTIC DEFORMATION OF PROTEIN}

\section{CHEMICAL STRUCTURE}

(Supplement to The Chemical Organization of Living Matter by C. F. Krafft)

Copyright 1938 by Carl F. Krafft
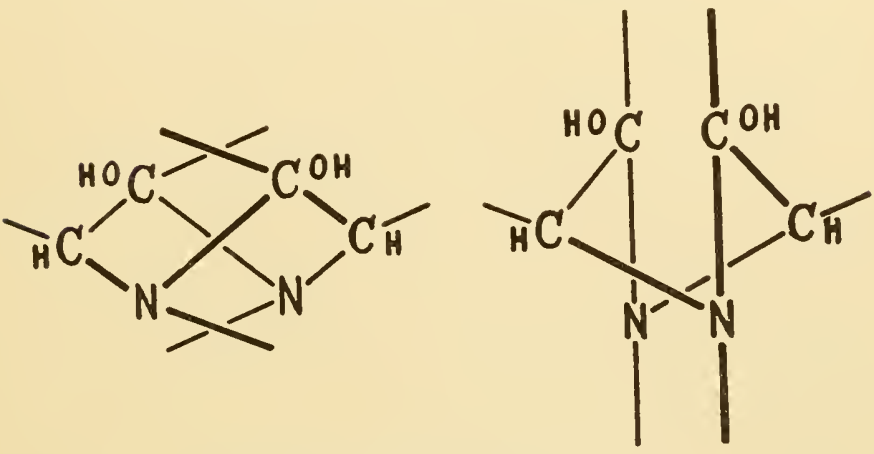

Double-strand polypeptide spiral

Wool fibers in an atmosphere of steam can be stretched elastically to twice their original length, which means that each molecule of the protein of which the fiber is composed must have this same elasticity. The chemical structure of the protein must therefore be such as to permit elastic stretching to twice its original length, but no more. The only explanation which has been recognized is that of W. T. Astbury, who assumes that the polypeptide chains of wool keratin and other proteins are ordinarily folded into diketopiperazine rings with intermediate - $\mathrm{CO}-\mathrm{CHR}-\mathrm{NH}$ - groups, but pass over into the simple zig-zag form when tension is applied. Notwithstanding its general acceptance, Astbury's explanation seems entirely inadequate. The change from the normal configuration (alpha-keratin) to the stretched configuration (beta-keratin) would have to result either from bending of the valence-bonds, or from swiveling about the same. If it results from bending, then we would have to assume that some of the valence-bonds can be bent elastically through 180 degrees, which is unusual 
to say the least. Furthermore if they were capable of such bending, then they would be brought into alignment when placed under tension, and not into a zig-zag configuration. On the other hand if it results from a swiveling of the atoms about their valence-bonds, then the sidechain connections to the surrounding structures would be destroyed during such swiveling movement, and furthermore there would be nothing to cause a return to the original configuration upon release of the tension.

In the double-strand spiral structure these difficulties are entirely eliminated. As the above diagrams clearly show, the spiral structure can be elastically stretched to exactly twice its original length without appreciably changing the positions of the hydroxyl groups by which it is anchored to adjacent spirals.

The spiral structure also accounts for the identity intervals of $3.5,4.5$, and 10 Angstroms along mutually perpendicular axes, such intervals having been determined experimentally for proteins (e.g., beta-keratin) in the stretched condition. Since the 4.5 and 10 Angstrom spacings have already been accounted for elsewhere, it remains to consider only the 3.5 Angstrom spacing. According to W. T. Astbury, this is supposed to be the length of one amino acid residue when stretched to the breaking-point. It will be the length of one such residue only as long as the valence-bonds remain at angles of 109 degrees, but there is an abundance of evidence that they can flex through moderate angles and are not rigidly fixed at 109 degrees. In order to account for the transition from alpha- to beta-keratin, Astbury assumes that the valence-bonds can flex elastically through angles of 180 degrees-although in order to account for the length of one amino acid residue, he assumes that they are incapable of any flexing whatever!

In the spiral structure shown in the above diagrams, the 3.5 Angstrom interval represents the distance between successive diketopiperazine rings while the system is under tension as shown in the diagram at the right. 



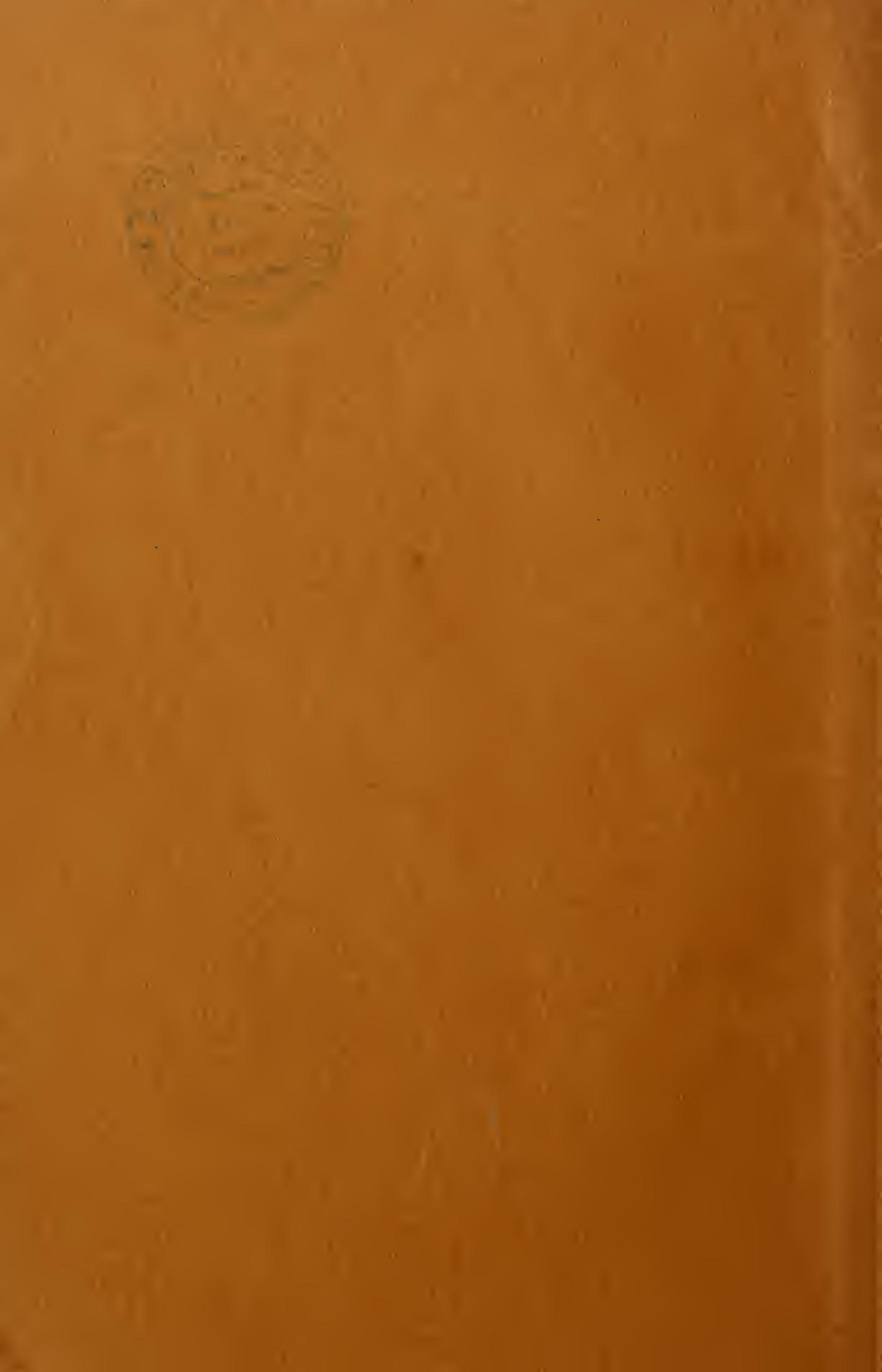

